\title{
Thermodynamic Equilibria in Carbon Nitride Photocatalyst Materials and Conditions for the Existence of Graphitic Carbon Nitride $\mathrm{g}-\mathrm{C}_{3} \mathrm{~N}_{4}$
}

Tiago Botari, ${ }^{\dagger, I l}$ William Paul Huhn, ${ }^{\dagger}$ Vincent Wing-hei Lau, ${ }^{\dagger}$ Bettina V. Lotsch, ${ }^{\dagger, \S_{\odot}}$ and Volker Blum ${ }^{*}, \odot$

${ }^{\dagger}$ Department of Mechanical Engineering and Materials Science, Duke University, Durham, North Carolina 27708, United States

${ }^{\ddagger}$ Max Planck Institute for Solid State Research, Heisenbergstraße 1, 70569 Stuttgart, Germany

${ }^{I I}$ Applied Physics Department, State University of Campinas, Campinas-SP 13083-970, Brazil

${ }^{\S}$ Department of Chemistry, University of Munich, Butenandtstraße 5-13, 81377 Munich, Germany

\author{
Supporting Information
}

ABSTRACT: We quantify the thermodynamic equilibrium conditions that govern the formation of crystalline heptazinebased carbon nitride materials, currently of enormous interest for photocatalytic applications including solar hydrogen evolution. Key phases studied include the monomeric phase melem, the 1D polymer melon, and the hypothetical hydrogenfree $2 \mathrm{D}$ graphitic carbon nitride phase " $\mathrm{g}-\mathrm{C}_{3} \mathrm{~N}_{4}$ ". Our study is based on density functional theory including van der Waals dispersion terms with different experimental conditions represented by the chemical potential of $\mathrm{NH}_{3}$. Graphitic carbon nitride is the subject of a vast number of studies, but its existence is still controversial. We show that typical conditions found in experiments pertain to the polymer melon (2D planes

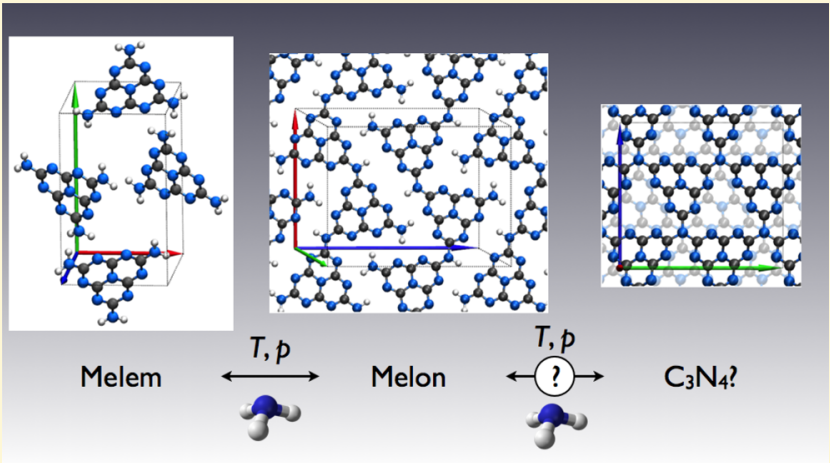
of $1 \mathrm{D}$ hydrogen-bonded polymer strands). In contrast, equilibrium synthesis of heptazine (h)-based g-h- $\mathrm{C}_{3} \mathrm{~N}_{4}$ below its experimentally known decomposition temperature requires much less likely conditions, equivalent to low $\mathrm{NH}_{3}$ partial pressures around $1 \mathrm{~Pa}$ at $500{ }^{\circ} \mathrm{C}$ and around $10^{3} \mathrm{~Pa}$ even at $700{ }^{\circ} \mathrm{C}$. $\mathrm{A}$ recently reported synthesis of triazine $(\mathrm{t})$-based $\mathrm{g}-\mathrm{t}-\mathrm{C}_{3} \mathrm{~N}_{4}$ in a salt melt is interpreted as a consequence of the altered local chemical environment of the $\mathrm{C}_{3} \mathrm{~N}_{4}$ nanocrystallites.

\section{INTRODUCTION}

Graphitic carbon nitride materials are subject to enormous recent attention as materials for catalysis, ${ }^{1}$ particularly for photocatalytic hydrogen evolution. ${ }^{2-5}$ Even though the first characterizations date back to at least the 1830 s, the nature and even the existence of completely $\mathrm{H}$-free crystalline polymeric $\mathrm{C}-\mathrm{N}$ compounds continue to be subject of intense debate. $^{7-9}$ Past synthesis and characterization efforts revealed triazine $^{8,9}(\mathrm{t}) \mathrm{C}_{3} \mathrm{~N}_{3}$ units and heptazine ${ }^{7}(\mathrm{~h}) \mathrm{C}_{6} \mathrm{~N}_{7}$ units as the most important building blocks. Some of the most important related compounds are illustrated in Figure 1:

(i) The $\mathrm{NH}_{2}$ terminated heptazine monomer melem (a), its dimer, a likely reaction intermediate (b), ${ }^{10}$ and the confirmed crystalline form of monomeric melem (c). ${ }^{11,12}$

(ii) Melon $(\mathrm{d}-\mathrm{f})$, a $1 \mathrm{D}$ heptazine polymer that crystallizes in two-dimensional (2D) hydrogen-bonded planes stacked threedimensionally in nanocrystalline platelets. ${ }^{13-15}$

(iii) $\mathrm{g}-\mathrm{t}-\mathrm{C}_{3} \mathrm{~N}_{4}(\mathrm{~g}-\mathrm{i})$, a possible periodic triazine-based equivalent of heptazine-based $\mathrm{g}-\mathrm{C}_{3} \mathrm{~N}_{4}{ }^{16-19}$ recently reported to have been successfully synthesized in a salt melt. ${ }^{8,9}$

(iv) g-h- $\mathrm{C}_{3} \mathrm{~N}_{4}(\mathrm{j}-\mathrm{l})$, a much discussed but still hypothetical hydrogen-free layered $\mathrm{C}-\mathrm{N}$ phase of heptazine units connected via triply bonded $\mathrm{N}$ atoms. ${ }^{20}$ This structure is often portrayed as $2 \mathrm{D}$ but exhibits a significant long-range corrugation in firstprinciples simulations ${ }^{21,22}$ accompanied by the bridging $\mathrm{N}$ atoms adopting a more $\mathrm{sp}^{3}$-like local geometry.

Remarkably, despite overwhelming follow-up attention to the seminal paper on $\mathrm{C}-\mathrm{N}$ compound-based water splitting, ${ }^{2}$ the basic (intrinsic) atomistic mechanisms behind the catalytic activity and how to control them remain poorly understood. Heptazine-based g-h- $\mathrm{C}_{3} \mathrm{~N}_{4}$, subject to a host of theoretical studies (e.g., refs 20 and 23-28), remains elusive in experiments. ${ }^{9,29}$ Note that g-h- $\mathrm{C}_{3} \mathrm{~N}_{4}$ was originally modeled as the $1 \mathrm{D}$ polymer melon, ${ }^{2}$ although a large number of subsequent papers used the fully condensed, stoichiometric $\mathrm{C}_{3} \mathrm{~N}_{4}$ in their discussion. For instance, out of 12 journal papers on $\mathrm{C}-\mathrm{N}$ materials ${ }^{30-41}$ that appeared on Google Scholar in a time frame of 3 weeks prior to submission of the present manuscript, five ${ }^{31,35-38}$ show images of g-h- $\mathrm{C}_{3} \mathrm{~N}_{4}$, five others use the term $\mathrm{g}-\mathrm{C}_{3} \mathrm{~N}_{4}$ as an apparent fact without further discussion of the atomic structure, ${ }^{32-34,39,40}$ and only two ${ }^{30,41}$ pay heed to the actual, more complex phase space of polymeric

Received: March 8, 2017

Revised: April 27, 2017

Published: April 28, 2017 


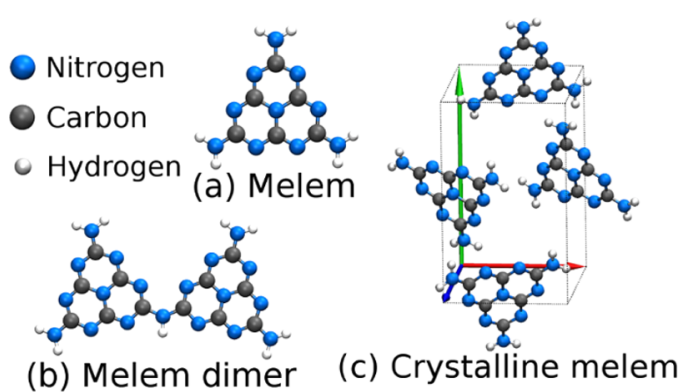

(b) Melem dimer

(c) Crystalline melem

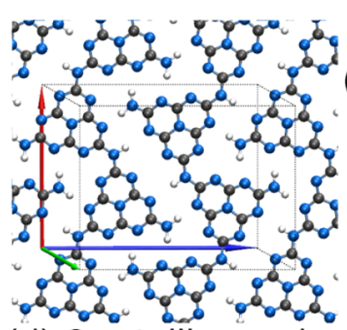

(d) Crystalline melon

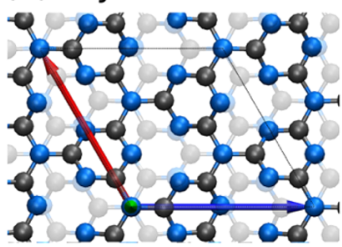

(g) $\mathrm{g}-\mathrm{t}-\mathrm{C}_{3} \mathrm{~N}_{4}$

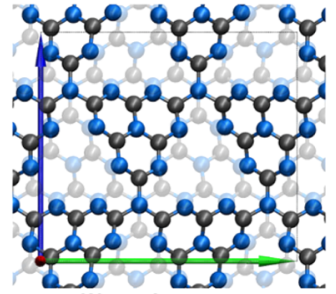

(j) g-h- $\mathrm{C}_{3} \mathrm{~N}_{4}$ (e) Crystalline melon Lateral view 1

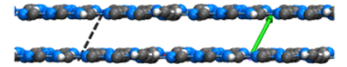

(f) Crystalline melon Lateral view 2

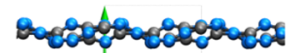

-

(h) g-t- $\mathrm{C}_{3} \mathrm{~N}_{4}$

Lateral view 1

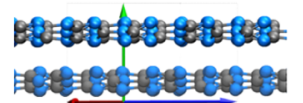

(i) g-t- $\mathrm{C}_{3} \mathrm{~N}_{4}$

Lateral view 2
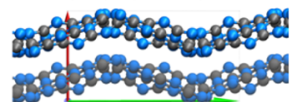

(k) g-h- $\mathrm{C}_{3} \mathrm{~N}_{4}$

Lateral view 1

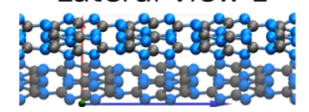

(l) g-h- $\mathrm{C}_{3} \mathrm{~N}_{4}$

Lateral view 2

Figure 1. Key $\mathrm{C}-\mathrm{N}$-based compounds considered in this work. (a) Melem molecule $\left(\mathrm{C}_{6} \mathrm{~N}_{7}\left(\mathrm{NH}_{2}\right)_{3}\right)$. (b) Melem dimer. ${ }^{10}$ (c) Unit cell of crystalline melem $^{11}$ (see Table S3). (d-f) Shifted single plane (SSP) stacked monoclinic model of the crystalline form of the 1D polymer melon (Table S4) viewed from three different angles. (g-i) Crystal structure model of g-t- $\mathrm{C}_{3} \mathrm{~N}_{4}$ viewed from three different angles (Table S7). (j-l) Crystal structure model of g-h- $\mathrm{C}_{3} \mathrm{~N}_{4}$ viewed from three different angles (Table S6). In subfigures $(\mathrm{g})$ and $(\mathrm{j})$, the second layer in each unit cell is shown lightly shaded.

carbon nitride. Instead, the (nano)crystalline phase reached during conventional syntheses ${ }^{7}$ appears to be melon. ${ }^{13}$ On the other hand, g-t $-\mathrm{C}_{3} \mathrm{~N}_{4}$ triazine (with the same formal composition as heptazine-based g-h- $\mathrm{C}_{3} \mathrm{~N}_{4}$ ) could be synthesized as reported recently ${ }^{8,9}$ but in a very different reaction environment. Here, the procedure used for the synthesis of the triazine-based g-t- $\mathrm{C}_{3} \mathrm{~N}_{4}$, employing a $\mathrm{LiCl} / \mathrm{KCl}$ salt melt, yields as the majority phase the lithium/chloride-intercalated poly(triazine imide) (PTI). ${ }^{42,43}$ Structurally, this is the incompletely condensed g-t $-\mathrm{C}_{3} \mathrm{~N}_{4}$, also discussed in ref 22 and shown in Figure S3, whereas the fully condensed g-t- $\mathrm{C}_{3} \mathrm{~N}_{4}$ appears to be a minor phase formed at the interface of the salt melt. ${ }^{43}$ If so, this formation process could be related to earlier work by chemical vapor deposition based on hydrogen-free, triazinebased precursors that were also reported to yield hydrogen-free g-t- $\mathrm{C}_{3} \mathrm{~N}_{4} \cdot{ }^{44,45}$ First-principles studies found that g-t- $\mathrm{C}_{3} \mathrm{~N}_{4}$ is less stable than the heptazine-based $\mathrm{g}-\mathrm{h}-\mathrm{C}_{3} \mathrm{~N}_{4}{ }^{20,23}$ Still, if the

triazine-based phase can be synthesized under some conditions, it is not clear why the heptazine-based phase cannot. In addition, even the H-containing crystalline phases, which can be made (crystalline melem and melon), show little or no activity for photochemical hydrogen evolution by themselves, ${ }^{10}$ supporting the idea that interfaces, disorder, defects, and/or accidentally inserted functionalities during synthesis play a major role in the observed activity. ${ }^{3,10}$ For instance, underreacted oligomeric melon species were implicated as potentially active, ${ }^{10}$ and specific chemical modifications can dramatically enhance this activity. ${ }^{46,47}$ In fact, melon obtained under certain synthesis conditions may well itself be an oligomeric phase.

The key motivation for this paper is to provide a quantitative understanding of the structure and relative stability of the basic $\mathrm{C}-\mathrm{N}$ phases under different reaction conditions, needed to arrive at a rational, reductionist understanding and control of the specific mechanisms governing activity. Specifically, we quantify the relative stability of the most important $\mathrm{C}-\mathrm{N}$ phases discussed in the literature by an ab initio thermodynamic approach. ${ }^{48}$ We deliberately restrict the analysis to the already large space of structure models discussed in the literature as a first perimeter, creating a sound base for future extensions, e.g., by including defects or targeted chemical modifications. A key result is that the heptazine-based $\mathrm{g}-\mathrm{C}_{3} \mathrm{~N}_{4}$ phase cannot be stable at conditions pertaining to conventional syntheses, at least not for the structures suggested in the literature so far. This observation emerges as a simple consequence of the thermodynamic equilibrium conditions connecting the different phases. Our approach is based on the fact that the different phases described above are all connected by simple reaction equilibria that involve the release of $\mathrm{NH}_{3}$. Therefore, the emergence of each phase at different conditions can be related to the $\mathrm{NH}_{3}$ chemical potential as the critical quantity that characterizes the reaction environment. Experimentally, species other than $\mathrm{NH}_{3}$ (such as $\mathrm{CNH}$ or $\mathrm{C}_{2} \mathrm{~N}_{2}$ ) are only released during transformations of $\mathrm{C}-\mathrm{N}$ compounds at high temperature. ${ }^{42}$ They are usually taken to be indicative of the materials' decomposition, justifying our focus on $\mathrm{NH}_{3}$ as the gas-phase species connecting different triazine- or heptazine-based phases. Our analysis also allows us to place oligomeric intermediates ${ }^{10}$ in the context of the confirmed stable phases, showing that they are indeed close to equilibrium and should be considered to explain the observed chemical stability range of melon.

\section{METHODS}

Background. The standard route to synthesize $\mathrm{C}-\mathrm{N}$ compounds is rather straightforward. ${ }^{7}$ A commercially available $\mathrm{C}-\mathrm{N}$-containing substance, e.g., melamine $\left(\mathrm{C}_{3} \mathrm{~N}_{3}\left(\mathrm{NH}_{2}\right)_{3}\right)$, is heated for an extended period of time at a defined temperature in an open ${ }^{10}$ or closed vessel. The synthesis can then be rationalized as a continued condensation during which $\mathrm{NH}_{3}$ units are removed and escape:

Melamine $\leftrightarrows$ melem (per heptazine unit)

$$
2 \mathrm{C}_{3} \mathrm{~N}_{3}\left(\mathrm{NH}_{2}\right)_{3} \rightleftarrows \mathrm{C}_{6} \mathrm{~N}_{7}\left(\mathrm{NH}_{2}\right)_{3}+2 \mathrm{NH}_{3}
$$

Melem $\leftrightarrows$ melon

$$
\begin{aligned}
& \mathrm{C}_{6} \mathrm{~N}_{7}\left(\mathrm{NH}_{2}\right)_{3} \rightleftarrows \mathrm{C}_{6} \mathrm{~N}_{7}(\mathrm{NH})\left(\mathrm{NH}_{2}\right)+\mathrm{NH}_{3} \\
& \text { Melon } \leftrightarrows \mathrm{g}-[\mathrm{t} / \mathrm{h}]-\mathrm{C}_{3} \mathrm{~N}_{4} \\
& \mathrm{C}_{6} \mathrm{~N}_{7}(\mathrm{NH})\left(\mathrm{NH}_{2}\right) \rightleftarrows \mathrm{C}_{6} \mathrm{~N}_{8}+\mathrm{NH}_{3}
\end{aligned}
$$

The formation of crystalline melem following eq 1 was reported at $T=$ $450{ }^{\circ} \mathrm{C}$ for $5 \mathrm{~h}$ in a closed ampule; ${ }^{11}$ other procedures in an open vessel have been reported, ${ }^{49}$ although we found that the product 
obtained contains higher condensates such as oligomers. ${ }^{46}$ Melon formation, eq 2, was reported for a range of conditions ${ }^{7}$ in opened (e.g., lidded crucible) or closed vessels (sealed quartz ampule), including $\mathrm{T}=490{ }^{\circ} \mathrm{C}$ for 4 days (opened vessel), ${ }^{50} \mathrm{~T}=500{ }^{\circ} \mathrm{C}$ for 15 $\mathrm{h}$ (opened vessel),$^{15} \mathrm{~T}=550{ }^{\circ} \mathrm{C}$ for $12 \mathrm{~h}$ (opened vessel), ${ }^{10}$ and $\mathrm{T}=$ $630{ }^{\circ} \mathrm{C}$ for $12 \mathrm{~h}$ (closed vessel). ${ }^{13} \mathrm{It}$ is worth noting that, because of the release of ammonia, these syntheses are often associated with rather high ammonia pressures. For instance, differential scanning calorimetry in ref 11 needed to be carried out in steel pressure crucibles "because conventional alumina crucibles burst due to evolution of ammonia," and pressures up to 12 bar are reported in salt-melt triazine-based g-t- $\mathrm{C}_{3} \mathrm{~N}_{4}$ synthesis. ${ }^{8}$

The resulting $\mathrm{C}-\mathrm{N}$-based materials are reported to decompose at temperatures around $\mathrm{T}=680-700{ }^{\circ} \mathrm{C} .{ }^{42}$ However, the formation of gaseous decomposition fragments other than $\mathrm{NH}_{3}$ has been observed at temperatures as low as $T=480{ }^{\circ} \mathrm{C}(\mathrm{CNH})$ and $T=550{ }^{\circ} \mathrm{C}$ $\left(\mathrm{C}_{2} \mathrm{~N}_{2}\right),{ }^{42}$ i.e., typical formation conditions of melon may already overlap with the onset of decomposition of some intermediate fractions of the material. Interestingly, well-defined crystalline phases of melamine-melem adducts have also been synthesized "at temperatures ranging from 350 to $400^{\circ} \mathrm{C}$ with reaction times of at least 7 days," ${ }^{51}$ potentially providing a lower-temperature limit to the formation range of crystalline melem. The corresponding structure models are shown in Figure 2. As mentioned above, however, the formation of g-h- $\mathrm{C}_{3} \mathrm{~N}_{4}$, eq 3 , has never to our knowledge been unambiguously confirmed in any synthesis.

Computational Approach. All first-principles calculations in this work were carried out using density-functional theory (DFT) including correction terms for long-range dispersion interactions. We employed the highly accurate ${ }^{52}$ all-electron electronic structure code FHI-aims ${ }^{53-55}$ with "tier2" basis sets and "tight" numerical settings.

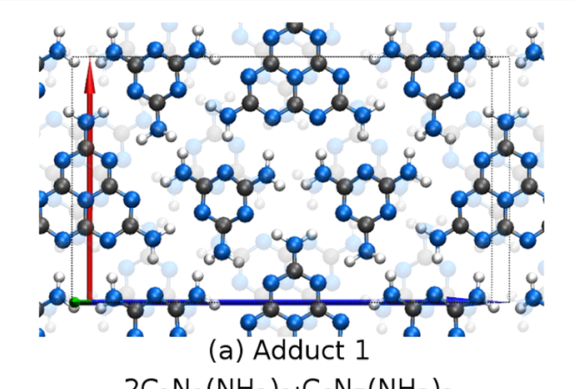

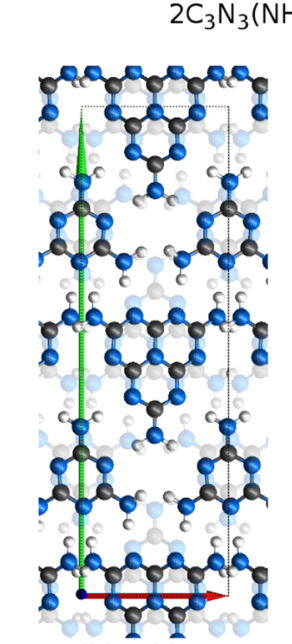

(b) Adduct 2

$\mathrm{C}_{3} \mathrm{~N}_{3}\left(\mathrm{NH}_{2}\right)_{3} \cdot \mathrm{C}_{6} \mathrm{~N}_{7}\left(\mathrm{NH}_{2}\right)_{3}$

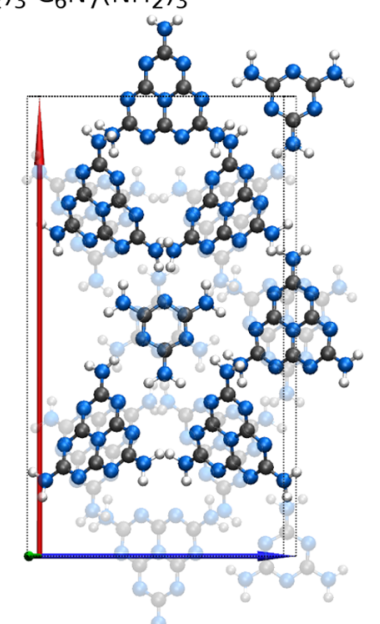

(c) Adduct 3
Figure 2. Crystal structure models of three melamine-melem adduct phases proposed in ref 51 using unit cell parameters and atomic positions determined by first-principles relaxations in this work (Table S7).
With these settings, conformational energy hierarchies of complex hydrogen-bonded systems are converged to a few $\mathrm{meV}$ per hydrogen bond. $^{53}$ All atomic positions and unit cell parameters were fully relaxed ${ }^{56}$ to local minima (residual energy gradients: $5 \times 10^{-3} \mathrm{eV} / \AA ̊$ or below) of the $\mathrm{PBE}^{57}$ semilocal density functional with the Tkatchenko-Scheffler $(\mathrm{TS})^{58}$ pairwise van der Waals correction. Because van der Waals bonding plays a significant role in these systems, we validated our key results against a recent, extensively benchmarked $^{59-61}$ many-body dispersion (MBD) ${ }^{62}$ approach. Furthermore, the PBE part of the functional was checked by substituting, for a few key compounds, the hybrid HSE06 functional ${ }^{63}$ in a recent linear-scaling periodic implementation ${ }^{64,65}$ using "intermediate" basis settings, as documented in Table S1. The corresponding density functionals are referred to as $\mathrm{PBE}+\mathrm{TS}, \mathrm{PBE}+\mathrm{MBD}$, and HSE06+MBD below.

For our analysis of the chemical equilibria between different $\mathrm{C}-\mathrm{N}$ phases, we surveyed and compared the different structure models put forth in the literature (experiment and theory), as well as different candidate structures of our own. Within DFT-PBE+TS, the key geometry parameters of the fully optimized structures (local minima of the potential energy surface) agree well with earlier experimental findings ${ }^{11,13-15}$ (see Supporting Information (SI) for details: Table S3 for crystalline melem, Tables S4 and S5 for data related to different models of melon, Table S6 for g-h- $\mathrm{C}_{3} \mathrm{~N}_{4}$, Table S7 for g-t- $\mathrm{C}_{3} \mathrm{~N}_{4}$, and Table S8 for melamine-melem adduct phases).

We can relate the formation equilibria (eqs $1-3$ ) to the temperature and $\mathrm{NH}_{3}$ partial pressure under reaction conditions using firstprinciples total energy calculations for all involved phases. The finitetemperature and pressure properties of $\mathrm{NH}_{3}$ are represented by chemical potential $\mu_{\mathrm{NH}_{3}}$. In a gas phase environment, $\mu_{\mathrm{NH}_{3}}$ can be related to the $\mathrm{NH}_{3}$ partial pressure $p_{\mathrm{NH}_{3}}$ and to $T$ using its experimentally known form $\mu_{\mathrm{NH}_{3}}(p, T)$. Specifically, we employ a parametrized ideal-gas expression for $\mu_{\mathrm{NH}_{3}}(p, T)$ (SI, eqs S1-S3 and Table S2), ${ }^{66}$ which is in excellent agreement with available thermochemical data in the literature ${ }^{67-69}$ (Figure S1). Importantly, because $\mu_{\mathrm{NH}_{3}}$ is an abstract quantity, it can be used to reflect any other near-equilibrium $\mathrm{NH}_{3}$ reservoir as well, including $\mathrm{NH}_{3}$ exchanged with and/or solvated in a salt melt. ${ }^{8}$

We can quantify the equilibria (eqs 1-3) in terms of their approximate Gibbs free energy differences based on the total energies of the lowest-energy structures of each phase referenced to the energy of a melem molecule and to $\mu_{\mathrm{NH}_{3}}$

$$
\begin{aligned}
& \Delta G^{\text {melem,cryst. }}=G^{\text {melem,cryst. }}-G^{\text {melem,mol. }} \\
& \Delta G^{\text {melon }}=G^{\text {melon }}-\left(G^{\text {melem,mol. }}-\mu_{\mathrm{NH}_{3}}\right) \\
& \Delta G^{\mathrm{g}-\mathrm{C}_{3} \mathrm{~N}_{4}}=G^{\mathrm{g}-\mathrm{C}_{3} \mathrm{~N}_{4}}-\left(G^{\text {melem,mol. }}-2 \mu_{\mathrm{NH}_{3}}\right)
\end{aligned}
$$

The energies $G^{\text {melem,cryst. }} G^{\text {melon }}$, and $G^{\mathrm{g}-\mathrm{C}_{3} \mathrm{~N}_{4}}$ are taken per unit of melem/heptazine in accordance with eqs 2 and 3. The subtracted energy of molecular melem, $G^{\text {melem,mol. }}$, thus acts as a common offset, leaving $\mu_{\mathrm{NH}_{3}}$ as the only experimental variable. Because all relevant phases except for $\mathrm{NH}_{3}$ are solids, by far the largest dependence on external conditions (temperature, pressure and partial pressures, $\mathrm{NH}_{3}$ reservoir) enters through $\mu_{\mathrm{NH}_{3}}$. We therefore approximate $G$ for all solid phases by their DFT-calculated total energies $E$, omitting their smaller temperature and pressure dependence (particularly the vibrational contribution). Likewise, effects of the reaction environment (gas-phase or salt melt) and kinetic effects (barriers and/or kinetically necessary intermediates) are not yet included. Although it is hard (if not impossible) to treat the latter factors conclusively from firstprinciples alone, they will be considered qualitatively in the discussion below. Finally, we note that synthesis paths are possible that start with precursors other than melamine, e.g., cyanamide, dicyanamide, urea, or thiourea. However, our analysis covers the thermodynamic (near)equilibrium regime. Thus, as long as the reaction leads to one of the intermediates of the governing reactions (eqs 1-3), i.e., melamine, 

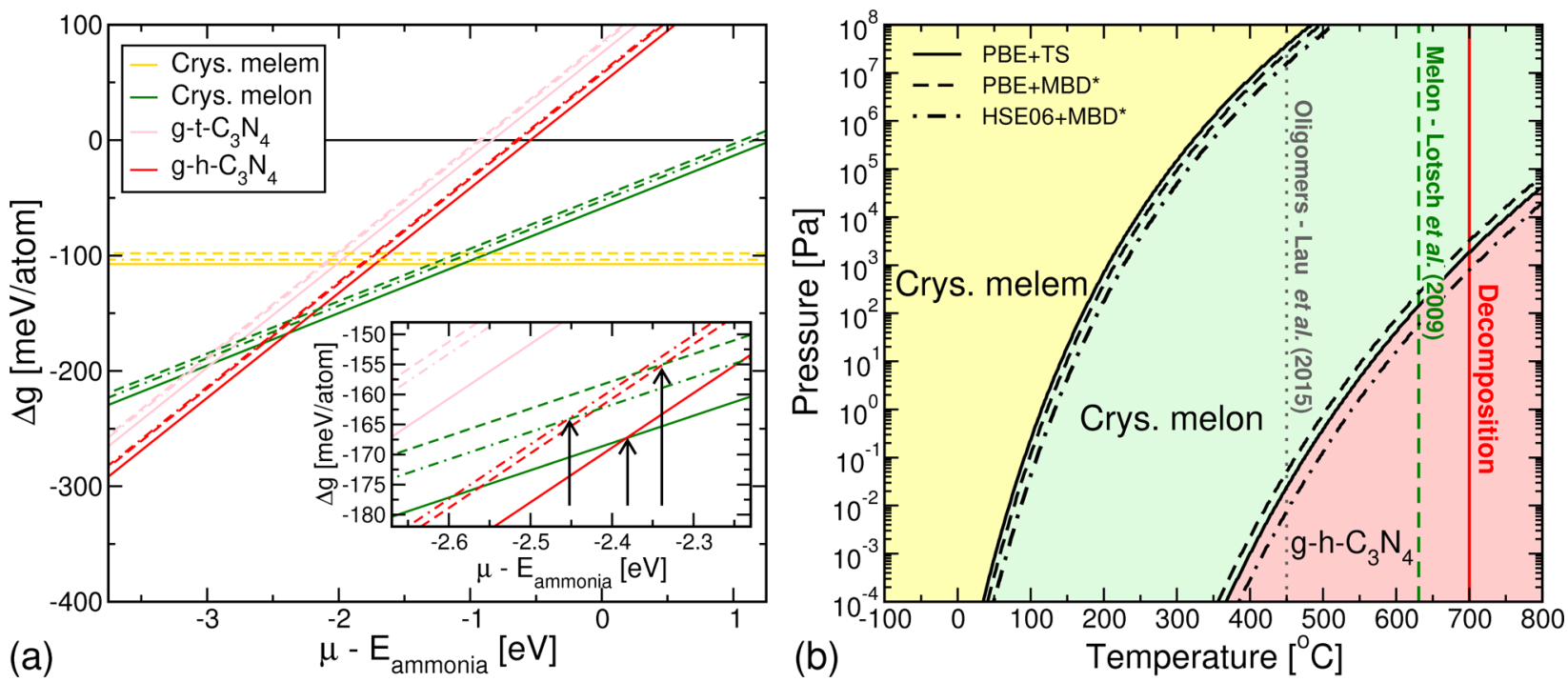

Figure 3. (a) Calculated Gibbs free energies per atom, $\Delta g$, for crystalline melem, melon (lowest-energy SSP stacked model, see Table S4), and the lowest-energy model of g-t- $\mathrm{C}_{3} \mathrm{~N}_{4}{ }^{22}$ and g-h- $\mathrm{C}_{3} \mathrm{~N} 4^{21,22}$ as a function of the ammonia chemical potential $\mu_{\mathrm{NH}_{3}}$. The isolated melem molecule is taken as the reference compound (zero line). Results are provided for three different density functionals, PBE+TS, PBE+MBD, and HSE06+MBD, for the lowest-energy geometries at the PBE+TS level of theory. (b) Calculated temperature $/ \mathrm{NH}_{3}$ partial pressure phase diagram based on $\mu_{\mathrm{NH}}(p, T)(\mathrm{SI}$, eqs S1-S3, Table S2, and Figure S1) comparing three phases: crystalline melem, crystalline melon, and g-h-C $\mathrm{N}^{4}$. Different colored areas indicate where a given phase has the lowest $\Delta g$. Phase boundaries are drawn for all three density functionals considered. Experimental conditions referenced are from refs 10 and 13 .

melem, melon, or g-h- $\mathrm{C}_{3} \mathrm{~N}_{4}$, our conclusions are independent of the starting point and kinetic paths to reach one of those products. We do, however, believe that different synthesis starting points may leave behind different defect moieties in the product that may affect its practical catalytic properties. We demonstrated the effect of such potential moieties, e.g., related to cyanamide or urea, in two different recent studies. ${ }^{46,47}$

\section{RESULTS AND DISCUSSION}

Structure of Phases Considered. For melon, the parameter that emerges from experimental XRD with the highest degree of certainty is the interplanar spacing $d=$ $3.19^{13}-3.26 \AA^{14,15}$ of successive 2D hydrogen-bonded planes characterized by a sharp powder diffraction peak. Although different authors agree on the in-plane arrangement of heptazine polymer strands in 3D melon, different stacking sequences of $2 \mathrm{D}$ planes have been put forward. ${ }^{13,15,70}$ Specifically, stacks of individual, laterally shifted planes (here referred to as "shifted single plane" (SSP) with monoclinic unit cells) and orthorhombic $A B$ stackings of pairs of planes are summarized in refs 15 and 70 . For the monoclinic SSP and orthorhombic $\mathrm{AB}$ structure models, we tested grids of different interplanar registries as starting points for full DFT-PBE+TS geometry optimizations. The lowest total energy per lateral melon unit cell (72 atoms) is obtained for SSP stacking (see Table S4 for structural details) with a predicted interplanar distance $d=3.20 \AA$. There are, however, several other energetically indistinguishable SSP-stacked local minimum structures. Furthermore, $\mathrm{AB}$-stacked geometries are not much higher in energy, i.e., $\Delta E \lesssim 0.10 \mathrm{eV}$ per unit cell or $\sim 1-2$ $\mathrm{meV} /$ atom for a model similar to that in ref 70 (Table S5).

The $A B$ stacked model remains nearly orthorhombic in our simulations. In Figure S2, we compare published experimental powder X-ray diffraction (PXRD) data with simulated PXRD data for the fully optimized structure models obtained in this work. There is considerable variation already between experimental data obtained from different syntheses. ${ }^{13,15}$ However, some features are reproduced in both experiments, for instance, the low-angle diffraction peak at around $2 \theta=13^{\circ}$, which indicates a slightly better match of the orthorhombic ABstacked computational model. One main finding from our analysis of SSP- and AB-stacked models of melon with different interplanar stacking relationships is that there are local energy minima with almost equivalent total energies in line with earlier analyses as well. ${ }^{71}$ It is thus likely that small local variations in experimental conditions would lead to locally different stacking relationships in different melon platelets. Shifts between successive planes with similar probabilities for different shift directions could also lead to an overall stacking that is more similar to the orthorhombic case and thus to the experimental PXRD. Because the total energies of the different low-energy melon models are similar ${ }^{71}$ and their stoichiometries are identical, this local structural difference will not affect the overall phase equilibria between stoichiometrically different phases examined in this work.

For the g-h- $\mathrm{C}_{3} \mathrm{~N}_{4}$ and g-t- $\mathrm{C}_{3} \mathrm{~N}_{4}$ phases, we considered planar arrangements as well as arrangements that allowed for longerranged corrugation, specifically, the significantly corrugated g-h$\mathrm{C}_{3} \mathrm{~N}_{4}$ and g-t $-\mathrm{C}_{3} \mathrm{~N}_{4}$ model of refs 21 and 22 . In our calculations (DFT-PBE+TS), these corrugated models are lower in total energy than essentially planar models by $44 \mathrm{meV} /$ atom (g-h$\mathrm{C}_{3} \mathrm{~N}_{4}$ ) and $54 \mathrm{meV} /$ atom $\left(\mathrm{g}-\mathrm{t}-\mathrm{C}_{3} \mathrm{~N}_{4}\right)$, i.e., by rather sizable amounts. In addition, there is a large space of further longrange corrugated models that could conceivably yield even lower energy structures, which we did not explore. However, it is clear that at least the idealized g-h- $\mathrm{C}_{3} \mathrm{~N}_{4}$ structure cannot be truly $2 \mathrm{D}$ in nature. The g-t- $\mathrm{C}_{3} \mathrm{~N}_{4}$ model of ref 22 remains somewhat more planar; although we tried a g-t- $\mathrm{C}_{3} \mathrm{~N}_{4}$ model with a similarly large long-range corrugation as the heptazinebased compound, for triazine, this larger corrugation did not yield a lower total energy than that of the model of ref 22 . Regarding the exact nature of the corrugation, the main total 

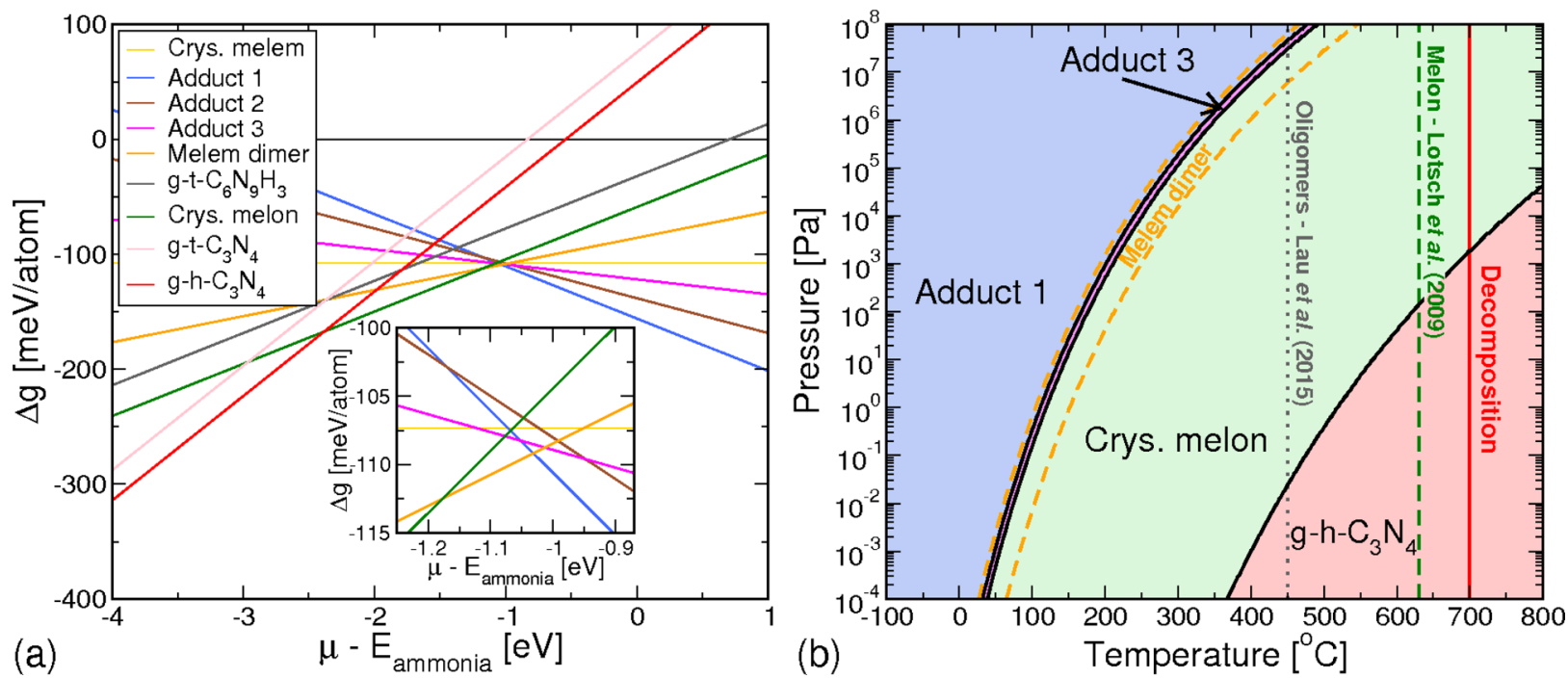

Figure 4. Gibbs free energies and phases as in Figure 3 but reflecting a wider space of potential intermediate phases. Results are provided for the PBE +TS density functional. (a) Calculated Gibbs free energies per atom, $\Delta g$, for the melamine-melem adduct structures 1-3 of Figure 2, crystalline melem, the lowest-energy SSP stacked model of melon, g-t- $\mathrm{C}_{3} \mathrm{~N}_{4}, \mathrm{~g}-\mathrm{h}-\mathrm{C}_{3} \mathrm{~N} 4$, and a hypothetical melem dimer phase as a function of the ammonia chemical potential $\mu_{\mathrm{NH}_{3}}$. Additionally, a suggested crystalline PTI compound g-t- $\mathrm{C}_{6} \mathrm{~N}_{9} \mathrm{H}_{3}{ }^{22}$ (see Figure S3 and Table S9), reportedly achieved in the chemical environment of a salt melt, ${ }^{8,9}$ is included but found to be unstable in the present analysis. The reference compound (zero line) is molecular melem. $\mu_{\mathrm{NH}_{3}}$ is given with reference to the calculated total energy per atom of an $\mathrm{NH}_{3}$ molecule. (b) Calculated temperature/ $\mathrm{NH}_{3}$ partial pressure phase diagram based on $\mu_{\mathrm{NH}_{3}}(p, T)$. In DFT-PBE+TS, the melamine-melem adduct phases 1 and 3 are slightly more stable than crystalline melem up to the stability range of melon. A hypothetical melem dimer phase could have a narrow stability range of its own. $T=450{ }^{\circ} \mathrm{C}$ is marked to indicate the melem oligomer synthesis temperature used in ref 10 .

energy $\operatorname{term}(\mathrm{s})$ associated with nonplanarity (compared to purely planar models) are likely already included in the structure models of refs 21 and 22, which we considered in our work.

Thermodynamic Equilibria. Figure 3 summarizes the predicted phase equilibria for the restricted space of the three key phases in the $\mathrm{C}-\mathrm{N}$ system and assesses their dependence on the level of theory used (PBE+TS, PBE+MBD, and HSE06+MBD). To clarify the essential trends related to melon and g-h- $\mathrm{C}_{3} \mathrm{~N}_{4}$, Figure 3(a) is limited to $\Delta g$ (i.e., $\Delta G$ / atom) for crystalline melem, crystalline melon (SSP stacked), and for the corrugated heptazine-based g-h- $\mathrm{C}_{3} \mathrm{~N}_{4}$ and triazinebased g-t- $\mathrm{C}_{3} \mathrm{~N}_{4}$ structure models as a function of $\mu_{\mathrm{NH}_{3}}$ and is evaluated at the PBE+TS equilibrium structures. Molecular melem is used as a common reference (energy zero). Compared to the energy scale that distinguishes different equilibrium phases in Figure 3(a), the differences between the different functionals cancel to a large extent, i.e., total-energy differences based on the PBE+TS functional provide a reliable description in this case. The transition between different phases is marked by the crossing points of different curves in Figure $3(\mathrm{a})$; the equilibrium $\mu_{\mathrm{NH}_{3}}$ varies by only $0.05 \mathrm{eV}$ (melem/ melon) and by $0.12 \mathrm{eV}$ (melon/g-h- $\mathrm{C}_{3} \mathrm{~N}_{4}$ ) between different functionals. g-t- $\mathrm{C}_{4} \mathrm{~N}_{3}$ is found to be higher in energy than g-h$\mathrm{C}_{3} \mathrm{~N}_{4}$ by $0.04 \mathrm{eV} /$ atom in both PBE+TS and PBE+MBD, a rather notable difference, although still in a range that could be overturned by changes to the chemical environment (such as intercalation of ions from a salt melt). The structures used reflect the lowest-energy known corrugated structure models for each phase, ${ }^{21,22}$ which are lower in energy by $\sim 0.04-0.06$ $\mathrm{eV} /$ atom than their smaller-scale less-corrugated counterparts (Tables S6 and S7). We note that, in principle, even lowerenergy structure models cannot be ruled out if a very different corrugated model for g-t- $\mathrm{C}_{4} \mathrm{~N}_{3}$ or g-h- $\mathrm{C}_{3} \mathrm{~N}_{4}$ could be found. Although our present work is restricted to relating existing models suggested in the literature to one another, the energy differences between the existing flat and corrugated models suggest that the resulting uncertainty will not exceed a few tens of $\mathrm{meV} /$ atom at most. We also note, however, that other extensive first-principles structure searches have targeted the more general space of $\mathrm{C}_{3} \mathrm{~N}_{4}$ compounds, for instance, the recent searches reported in refs 72 and 73 . These studies also cover different, denser, three-dimensionally bonded $\mathrm{C}_{3} \mathrm{~N}_{4}$ phases that could become stable at much higher hydrostatic pressure (not the same as the partial pressure of ammonia, the relevant quantity in our present work). However, for the pressure range of interest in the present work, these composition-specific structure searches also do not reveal any significant lower-energy structural alternatives to the corrugated g-h- $\mathrm{C}_{3} \mathrm{~N}_{4}$ structure considered in our work. To verify this finding, we recomputed fully optimized structures and DFT$\mathrm{PBE}+\mathrm{TS}$ total energies for the $\mathrm{C}_{3} \mathrm{~N}_{4}$ structures reported in Table 1 of the Supporting Information accompanying the paper by Pickard et al., ${ }^{73}$ confirming that no significantly lower-energy compounds than $\mathrm{g}-\mathrm{h}-\mathrm{C}_{3} \mathrm{~N}_{4}$ were found. Details are given in Table S11 and the accompanying text in the SI of the present work.

Using the known shape of $\mu_{\mathrm{NH}_{3}}(p, T)$ for gas-phase ammonia and assuming a direct or indirect equilibrium with gas-phase $\mu_{\mathrm{NH}_{3}}(p, T)$, Figure 3(a) can be converted to an $\mathrm{NH}_{3}$ temperature-pressure phase diagram, shown in Figure 3(b). In the restricted space of phases considered in Figure 3(a), the key finding is the broad stability area of melon for common synthesis temperatures and even rather low ammonia partial pressures, consistent with the literature. $\mathrm{g}-\mathrm{h}-\mathrm{C}_{3} \mathrm{~N}_{4}$, on the other hand, cannot become the stable phase even at very high 
synthesis temperatures unless the $\mathrm{NH}_{3}$ partial pressure in the system is very low. For example, $p_{\mathrm{NH}_{3}}<50 \mathrm{~Pa}$ would have to be maintained so that $\mathrm{g}-\mathrm{C}_{3} \mathrm{~N}_{4}$ could form at $T \approx 600{ }^{\circ} \mathrm{C}$, a temperature very close to the known overall decomposition temperature of the material. ${ }^{42}$ Even lower $p$ would be required at lower temperatures. The common synthesis route-by pyrolysis in an atmosphere that produces $\mathrm{NH}_{3}$ and thus encourages a non-negligible $\mathrm{NH}_{3}$ partial pressure under all circumstances-is thus an impractical if not impossible synthesis path for g-h- $\mathrm{C}_{3} \mathrm{~N}_{4}$. For common synthesis conditions, particularly in a closed ampule, our calculations based on the presently known $\mathrm{C}-\mathrm{N}$ compound structure models thus rule out an effective synthesis of g-h- $\mathrm{C}_{3} \mathrm{~N}_{4}$. Only a different system geometry, much lower in total energy than even the heavily corrugated geometries considered in the literature so far, ${ }^{21,22}$ could change this picture. On the basis of our present findings, the only viable pathways to $\mathrm{H}$-free g-h- $\mathrm{C}_{3} \mathrm{~N}_{4}$ would require very low ammonia partial pressures. This could, perhaps, be achieved by synthesis in a very low-pressure environment, such as a dynamically pumped vacuum, assuming that other degradation paths of the material can be avoided.

The overall melon synthesis conditions reported in the literature $\left(T \approx 500-630{ }^{\circ} \mathrm{C}^{10,13,15,51}\right)$ agree well with the melon stability range identified in Figure $3(\mathrm{~b})$ at reasonable $\mathrm{NH}_{3}$ partial pressures of $10^{3}-10^{6} \mathrm{~Pa}$. In particular, the apparent difficulty of synthesizing g-h- $\mathrm{C}_{3} \mathrm{~N}_{4}$ at high temperatures, below the overall decomposition temperature of $\mathrm{C}-\mathrm{N}$ materials, is consistent with the extent of the predicted stability range of melon. However, the low-temperature limit of melon stability, bounded by melem-like compounds, is also of interest, not least because of the potential existence of oligomeric melem species in this range with greater activity than that of crystalline melon. ${ }^{10}$

In Figure 4, we refine the schematic picture given in Figure 3 by adding additional $\mathrm{C}-\mathrm{N}$ compound phases, including $\mathrm{H}$, that have been reported in the literature. Because all three levels of theory considered in this work were shown to be nearly equivalent above, we only show the PBE-TS level of theory in Figure 4; Figure S4 shows that practically no changes occur if PBE+MBD is used instead.

First, we turn to the three crystalline melamine-melem "adduct" phases reported in the literature ${ }^{51}$ and shown in Figure 2. Interestingly (inset of Figure 4a), the enlarged phase space shows that crystalline melem, crystalline melon, and the three adduct phases all cross in a narrow chemical potential range very close to one another with estimated Gibbs free energies only a few $\mathrm{meV} /$ atom apart. Regarding the lowestenergy phases at the PBE+TS level of theory, the "Adduct 1" and "Adduct 3" phases could be thermodynamically stable according to Figure 4a, whereas both "Adduct 2" and crystalline melem would remain narrowly unstable. However, for the low energy differences at issue here, either kinetic factors or entropic terms (vibrations or phonons, which were not considered in this work) or both may easily change the stability balance, rendering the calculated energetic balance of all four phases (“Adducts 1-3" and crystalline melem) consistent with past experimental observations. As a further key point, neither crystalline phase impacts the observed stability range of melon in a significant way.

Second, we consider the approximate stability of experimentally observed melem oligomer intermediates that have been shown to occur as synthesis intermediates. ${ }^{10}$ Crystal structures (if they exist) for oligomeric melem are not known, such that a precise total energy assessment on equal footing with the remaining compounds covered in Figure 4 is not possible. We can, however, look at related compounds to arrive at a lower limit estimate of the total energy of a melem oligomer. In Figure 4, we focus on the melem dimer (see Figure 1b) as the simplest example. To estimate the energy of a melem dimer embedded in a solid phase, we take the calculated total energy of isolated dimerized melem and add the known energy gain (per atom) of crystal formation from isolated monomeric melem. A solid orange line in Figure $4 \mathrm{a}$ and dashed orange lines in Figure $4 \mathrm{~b}$ indicate the relative energy and stability range of a melem dimer in this approximation. The approximate temperature/pressure range of melem dimer formation, calculated in this way, includes a temperature range from slightly below the crossover line between crystalline "Adduct 3 " and crystalline melon to approximately $50{ }^{\circ} \mathrm{C}$ higher than that line. Oligomers beyond dimers are expected to extend to somewhat higher temperatures, qualitatively well in line with the experimentally found synthesis conditions of previously observed melem oligomers. $^{10}$

We note that additional, high-pressure $\mathrm{C}-\mathrm{N}$-based compounds have not just been predicted computationally ${ }^{72,73}$ but that a H-containing, tetrahedrally bonded phase of composition $\mathrm{C}_{2} \mathrm{~N}_{3} \mathrm{H}$ (same composition as melon) with a defective wurtzite structure has also been synthesized experimentally at very high hydrostatic pressure and temperature $(p>27 \mathrm{GPa}, T>1500$ $\left.{ }^{\circ} \mathrm{C}\right) .{ }^{74,75}$ Although these conditions are outside the range covered in our phase diagrams (Figures 3 and 4), the existence of this and potentially other similar phases is entirely consistent with our findings.

A final crystal structure suggested in the literature for PTI, ${ }^{22,43}$ labeled g-t- $\mathrm{C}_{6} \mathrm{~N}_{9} \mathrm{H}_{3}$ (same stoichiometry as melon, see Figure S3 and Table S9), is included in Figure 4a for completeness but is not energetically competitive.

Evidently, triazine-based crystalline g-t- $\mathrm{C}_{3} \mathrm{~N}_{4}$ cannot appear as a stable phase in Figures 3 and 4 because its total energy is found to be above that of the heptazine-based compound g-h$\mathrm{C}_{3} \mathrm{~N}_{4}$. However, the triazine-based compound can apparently be synthesized, albeit under the very different conditions of a salt melt under high ammonia pressure and $\mathrm{T}=600{ }^{\circ} \mathrm{C} .{ }^{8}$ This g-t- $\mathrm{C}_{3} \mathrm{~N}_{4}$ formation is reported to occur in equilibrium with a high-pressure $\mathrm{NH}_{3}$ gas phase in an ampule at $600{ }^{\circ} \mathrm{C},{ }^{8}$ i.e., fundamentally the same thermodynamic conditions as considered in our work. We note that the $\mathrm{NH}_{3}$ chemical potential itself is well-defined by way of the equilibrium with its gas phase. Unless a very different triazine-based structure than the one considered here and in past works exists, it seems unlikely that this relatively large discrepancy could be explained by uncertainties in the level of theory used (different levels of DFT or temperature and pressure dependence of $\Delta G$ of the solid phases). The observed stability of g-t- $\mathrm{C}_{3} \mathrm{~N}_{4}$, if correct, would thus have to be related to the immediate environment of a salt melt, introducing ions in close proximity to or perhaps even intercalated into the $\mathrm{C}-\mathrm{N}$ compound formed. ${ }^{43}$ The chemical difference between a $\mathrm{H} / \mathrm{C} / \mathrm{N}$-rich environment (gas phase) and a hot salt melt environment is likely drastic and could well destabilize heptazine units completely, as is observed in the formation of triazine-based g-t- $\mathrm{C}_{3} \mathrm{~N}_{4}$. A detailed theoretical assessment of this environment, however, would not be easy and is certainly beyond the confines of the current work. 


\section{CONCLUSIONS}

In conclusion, our calculations show that crystalline, heptazinebased graphitic carbon nitride $\left(\mathrm{g}-\mathrm{h}-\mathrm{C}_{3} \mathrm{~N}_{4}\right)$ cannot be stable over melon under common synthesis conditions (e.g., contact with a high-pressure $\mathrm{NH}_{3}$ phase in a closed ampule at $T=600-700$ ${ }^{\circ} \mathrm{C}$ ) below the experimentally observed decomposition range of $\mathrm{C}-\mathrm{N}$-based materials. Extremely low $\mathrm{NH}_{3}$ partial pressures approaching the high-vacuum range would have to be guaranteed to reach $\mathrm{g}-\mathrm{h}-\mathrm{C}_{3} \mathrm{~N}_{4}$ below the decomposition temperature by a conventional synthesis approach. Because the recent observation of triazine-based g-t- $\mathrm{C}_{3} \mathrm{~N}_{4}$ is not in line with theoretical stability estimates of crystals of both phases and because this synthesis also happens in indirect contact with a high-pressure $\mathrm{NH}_{3}$ phase in a closed ampule, we speculate that this occurrence of a hydrogen-free $\mathrm{C}-\mathrm{N}$ is because of the contact with the very different chemical environment of a hot salt melt. The dominant phase(s) formed under common reaction conditions should be $\mathrm{H}$-containing, $\mathrm{NH}$-bridged heptazine-based phases like melon, the proper starting point for further atomistic studies of this class of materials, and its activity.

\section{ASSOCIATED CONTENT}

\section{S Supporting Information}

The Supporting Information is available free of charge on the ACS Publications website at DOI: 10.1021/acs.chemmater.7b00965.

Details of the computational settings used for the firstprinciples calculations and complete chemical potential expressions used for $\mathrm{NH}_{3}$; structure models for all $\mathrm{C}-\mathrm{N}$ based materials discussed in this paper; unit cells and optimized atomic coordinates at the DFT-PBE+TS level of theory for the atomic structure models of all major phases discussed in this work; these structures, as well as associated input and output files of the FHI-aims code, can also be found as ASCII files at the NOMAD repository with the permanent digital object identifier DOI: $10.17172 / \mathrm{NOMAD} / 2017.04 .20-1$ (PDF)

\section{AUTHOR INFORMATION}

Corresponding Author

*E-mail: volker.blum@duke.edu.

ORCID

Bettina V. Lotsch: 0000-0002-3094-303X

Volker Blum: 0000-0001-8660-7230

Notes

The authors declare no competing financial interest.

\section{ACKNOWLEDGMENTS}

T.B. acknowledges support by Grant 2014/11986-7, São Paulo Research Foundation (FAPESP). Support from a seed grant by Duke University's Energy Initiative is gratefully acknowledged. B.V.L. thanks the Deutsche Forschungsgemeinschaft (Grant LO1801/1-1) for financial support.

\section{REFERENCES}

(1) Zhu, J.; Xiao, P.; Li, H.; Carabineiro, S. A. C. Graphitic Carbon Nitride: Synthesis, Properties, and Applications in Catalysis. ACS Appl. Mater. Interfaces 2014, 6, 16449-16465.

(2) Wang, X.; Maeda, K.; Thomas, A.; Takanabe, K.; Xin, G.; Carlsson, J. M.; Domen, K.; Antonietti, M. A metal-free polymeric photocatalyst for hydrogen production from water under visible light. Nat. Mater. 2009, 8, 76-80.

(3) Wang, Y.; Wang, X.; Antonietti, M. Polymeric Graphitic Carbon Nitride as a Heterogeneous Organocatalyst: From Photochemistry to Multipurpose Catalysis to Sustainable Chemistry. Angew. Chem., Int. Ed. 2012, 51, 68-89.

(4) Marschall, R. Semiconductor Composites: Strategies for Enhancing Charge Carrier Separation to Improve Photocatalytic Activity. Adv. Funct. Mater. 2014, 24, 2421-2440.

(5) Liu, J.; Liu, Y.; Liu, N.; Han, Y.; Zhang, X.; Huang, H.; Lifshitz, Y.; Lee, S.-T.; Zhong, J.; Kang, Z. Metal-free efficient photocatalyst for stable visible water splitting via a two-electron pathway. Science 2015, 347, 970-974.

(6) von Liebig, J. On some nitrogen compounds. Annalen der Pharmacie 1834, 10, 1-47.

(7) Schwarzer, A.; Saplinova, T.; Kroke, E. Tri-s-triazines (sheptazines)-From a "mystery molecule" to industrially relevant carbon nitride materials. Coord. Chem. Rev. 2013, 257, 2032-2062.

(8) Algara-Siller, G.; Severin, N.; Chong, S. Y.; Björkman, T.; Palgrave, R. G.; Laybourn, A.; Antonietti, M.; Khimyak, Y. Z.; Krasheninnikov, A. V.; Rabe, J. P.; Kaiser, U.; Cooper, A. I.; Thomas, A.; Bojdys, M. J. Triazine-Based Graphitic Carbon Nitride: a TwoDimensional Semiconductor. Angew. Chem. Int. Ed. 2014, 53, 74507455.

(9) Kroke, E. gt-C 3N 4-The First Stable Binary Carbon(IV) Nitride. Angew. Chem. Int. Ed. 2014, 53, 11134-11136.

(10) Lau, V. W.-h.; Mesch, M. B.; Duppel, V.; Blum, V.; Senker, J.; Lotsch, B. V. Low-Molecular-Weight Carbon Nitrides for Solar Hydrogen Evolution. J. Am. Chem. Soc. 2015, 137, 1064-1072.

(11) Jürgens, B.; Irran, E.; Senker, J.; Kroll, P.; Müller, H.; Schnick, W. Melem (2,5,8-Triamino-tri-s-triazine), an Important Intermediate during Condensation of Melamine Rings to Graphitic Carbon Nitride: Synthesis, Structure Determination by X-ray Powder Diffractometry, Solid-State NMR, and Theoretical Studies. J. Am. Chem. Soc. 2003, $125,10288-10300$.

(12) Sattler, A.; Schnick, W. Zur Kenntnis der Kristallstruktur von Melem C6N7(NH2)3. Z. Anorg. Allg. Chem. 2006, 632, 238-242.

(13) Lotsch, B. V.; Döblinger, M.; Sehnert, J.; Seyfarth, L.; Senker, J.; Oeckler, O.; Schnick, W. Unmasking Melon by a Complementary Approach Employing Electron Diffraction, Solid-State NMR Spectroscopy, and Theoretical Calculations-Structural Characterization of a Carbon Nitride Polymer. Chem. - Eur. J. 2007, 13, 4969-4980.

(14) Tyborski, T.; Merschjann, C.; Orthmann, S.; Yang, F.; LuxSteiner, M. C.; Schedel-Niedrig, T. Crystal structure of polymeric carbon nitride and the determination of its process-temperatureinduced modifications. J. Phys.: Condens. Matter 2013, 25, 395402.

(15) Fina, F.; Callear, S. K.; Carins, G. M.; Irvine, J. T. S. Structural Investigation of Graphitic Carbon Nitride via XRD and Neutron Diffraction. Chem. Mater. 2015, 27, 2612-2618.

(16) Teter, D. M.; Hemley, R. J. Low-Compressibility Carbon Nitrides. Science 1996, 271, 53-55.

(17) Ortega, J.; Sankey, O. F. Relative stability of hexagonal and planar structures of hypothetical C3N4 solids. Phys. Rev. B: Condens. Matter Mater. Phys. 1995, 51, 2624-2627.

(18) Alves, I.; Demazeau, G.; Tanguy, B.; Weill, F. On a new model of the graphitic form of C3N4. Solid State Commun. 1999, 109, 697701.

(19) Lowther, J. E. Relative stability of some possible phases of graphitic carbon nitride. Phys. Rev. B: Condens. Matter Mater. Phys. 1999, 59, 11683-11686.

(20) Kroke, E.; Schwarz, M.; Horath-Bordon, E.; Kroll, P.; Noll, B.; Norman, A. D. Tri-s-triazine derivatives. Part I. From trichloro-tri-striazine to graphitic C3N4 structures. New J. Chem. 2002, 26, 508512.

(21) Gracia, J.; Kroll, P. Corrugated layered heptazine-based carbon nitride: the lowest energy modifications of $\mathrm{C} 3 \mathrm{~N} 4$ ground state. J. Mater. Chem. 2009, 19, 3013-3019.

(22) Melissen, S.; le Bahers, T.; Steinmann, S. N.; Sautet, P. Relationship between Carbon Nitride Structure and Exciton Binding 
Energies: A DFT Perspective. J. Phys. Chem. C 2015, 119, 2518825196.

(23) Huda, M. N.; Turner, J. A. Morphology-dependent optical absorption and conduction properties of photoelectrochemical photocatalysts for $\mathrm{H}_{2}$ production: A case study. J. Appl. Phys. 2010, 107, 123703.

(24) Guo, Y.; Yang, J.; Chu, S.; Kong, F.; Luo, L.; Wang, Y.; Zou, Z. Theoretical and experimental study on narrowing the band gap of carbon nitride photocatalyst by coupling a wide gap molecule. Chem. Phys. Lett. 2012, 550, 175-180.

(25) Xu, Y.; Gao, S.-P. Band gap of $\mathrm{C}_{3} \mathrm{~N}_{4}$ in the GW approximation. Int. J. Hydrogen Energy 2012, 37, 11072-11080.

(26) Du, A.; Sanvito, S.; Li, Z.; Wang, D.; Jiao, Y.; Liao, T.; Sun, Q.; Ng, Y. H.; Zhu, Z.; Amal, R; Smith, S. C. Hybrid Graphene and Graphitic Carbon Nitride Nanocomposite: Gap Opening, ElectronHole Puddle, Interfacial Charge Transfer, and Enhanced Visible Light Response. J. Am. Chem. Soc. 2012, 134, 4393-4397.

(27) Butchosa, C.; Guiglion, P.; Zwijnenburg, M. A. Carbon Nitride Photocatalysts for Water Splitting: A Computational Perspective. J. Phys. Chem. C 2014, 118, 24833-24842.

(28) Ruan, L.-W.; Zhu, Y.-J.; Qiu, L.-G.; Yuan, Y.-P.; Lu, Y.-X. First principles calculations of the pressure affection to g-C3N4. Comput. Mater. Sci. 2014, 91, 258-265.

(29) Mansor, N.; Miller, T. S.; Dedigama, I.; Jorge, A. B.; Jia, J.; Brazdova, V.; Mattevi, C.; Gibbs, C.; Hodgson, D.; Shearing, P. R.; Howard, C. A.; Cora, F.; Shaffer, M.; Brett, D. J.; McMillan, P. F. Graphitic Carbon Nitride as a Catalyst Support in Fuel Cells and Electrolyzers. Electrochim. Acta 2016, 222, 44-57.

(30) Yang, P.; Ou, H.; Fang, Y.; Wang, X. A Facile Steam Reforming Strategy to Delaminate Layered Carbon Nitride Semiconductors for Photoredox Catalysis. Angew. Chem. Int. Ed. 2017, 56, 3992-3996.

(31) Xu, X.; Liu, Y.; Zhu, Y.; Fan, X.; Li, Y.; Zhang, F.; Zhang, G.; Peng, W. Fabrication of a Cu2O/g-C3N4/WS2 Triple-Layer Photocathode for Photoelectrochemical Hydrogen Evolution. ChemElectroChem 2017, DOI: 10.1002/celc.201700014.

(32) Yuan, Y.; Huang, G.-F.; Hu, W.-Y.; Xiong, D.-N.; Zhou, B.-X.; Chang, S.; Huang, W.-Q. Construction of g-C3N4/CeO2/ZnO ternary photocatalysts with enhanced photocatalytic performance. $J$. Phys. Chem. Solids 2017, 106, 1-9.

(33) Zhang, Q.; Wang, H.; Chen, S.; Su, Y.; Quan, X. Threedimensional $\mathrm{TiO} 2$ nanotube arrays combined with g-C $3 \mathrm{~N} 4$ quantum dots for visible light-driven photocatalytic hydrogen production. RSC Adv. 2017, 7, 13223-13227.

(34) Liu, J.; Zhao, D.; Li, L.; Weng, M.; Zhang, C.; Zhang, S.; Zhu, J.; Feng, Y.; Shih, K.; Huang, W. Rational Design of Mini-size Carbon Nitride Nanosheets with Double Excitation- and pH-Dependent Fluorescence Behaviors for Two-photon Cell Imaging. Chem. - Asian J. 2017, 12, 835 .

(35) Ong, W.-J.; Putri, L. K.; Tan, Y.-C.; Tan, L.-L.; Li, N.; Ng, Y. H.; Wen, X.; Chai, S.-P. Unravelling charge carrier dynamics in protonated g-C3N4 interfaced with carbon nanodots as co-catalysts toward enhanced photocatalytic $\mathrm{CO} 2$ reduction: A combined experimental and first-principles DFT study. Nano Res. 2017, 10, 1-24.

(36) Li, S.; Wang, Z.; Wang, X.; Sun, F.; Gao, K.; Hao, N.; Zhang, Z.; Ma, Z.; Li, H.; Huang, X.; Huang, W. Orientation controlled preparation of nanoporous carbon nitride fibers and related composite for gas sensing under ambient conditions. Nano Res. 2017, 10, 17101719.

(37) Liu, Q.; Peng, Y.-J.; Xu, J.-C.; Ma, C.; Li, L.; Mao, C.-J.; Zhu, J.-J. Label-free electrochemiluminescence aptasensor for highly sensitive detection of acetylcholinesterase based on Au nanoparticles functionalized g-C3N4 nanohybrid. ChemElectroChem 2017, DOI: 10.1002/ celc. 201700035 .

(38) Li, J.-X.; Ye, C.; Li, X.-B.; Li, Z.-J.; Gao, X.-W.; Chen, B.; Tung, C.-H.; Wu, L.-Z. A Redox Shuttle Accelerates O2 Evolution of Photocatalysts Formed In Situ under Visible Light. Adv. Mater. 2017, 1606009.

(39) Zhu, C.; Jiang, Z.; Chen, L.; Qian, K.; Xie, J. L-cysteine-assisted synthesis of hierarchical NiS2 hollow spheres supported carbon nitride as photocatalysts with enhanced lifetime. Nanotechnology 2017, 28, 115708 .

(40) Vo, V.; Thi, X. D. N.; Jin, Y.-S.; Thi, G. L.; Nguyen, T. T.; Duong, T. Q.; Kim, S.-J. SnO2 nanosheets/g-C3N4 composite with improved lithium storage capabilities. Chem. Phys. Lett. 2017, 674, 4247.

(41) Savateev, A.; Pronkin, S.; Willinger, M. G.; Antonietti, M.; Dontsova, D. Towards Organic Zeolites and Inclusion Catalysts: Heptazine Imide Salts Can Exchange Metal Cations in the Solid State. Chem. - Asian J. 2017, 1.

(42) Bojdys, M. J. On new allotropes and nanostructures of carbon nitrides. Ph.D. thesis, Universität Potsdam, Potsdam, Germany, 2009.

(43) Wirnhier, E.; Döblinger, M.; Gunzelmann, D.; Senker, J.; Lotsch, B. V.; Schnick, W. Poly(triazine imide) with intercalation of lithium and chloride ions [(C3N3)2( $\mathrm{NH}(\mathrm{x}) \mathrm{Li}(1-\mathrm{x})) 3 \cdot \mathrm{LiCl}]:$ a crystalline 2D carbon nitride network. Chem. - Eur. J. 2011, 17, 3213-21.

(44) Kouvetakis, J.; Todd, M.; Wilkens, B.; Bandari, A.; Cave, N. Novel Synthetic Routes to Carbon-Nitrogen Thin Films. Chem. Mater. 1994, 6, 811-814.

(45) Todd, M.; Kouvetakis, J.; Groy, T. L.; Chandrasekhar, D.; Smith, D. J.; Deal, P. W. Novel Synthetic Routes to Carbon Nitride. Chem. Mater. 1995, 7, 1422-1426.

(46) Lau, V. W.-h.; Moudrakovski, I.; Botari, T.; Weinberger, S.; Mesch, M. B.; Duppel, V.; Senker, J.; Blum, V.; Lotsch, B. V. Rational design of carbon nitride photocatalysts by identification of cyanamide defects as catalytically relevant sites. Nat. Commun. 2016, 7, 12165.

(47) Lau, V. W.-h.; Yu, V. W.-z.; Ehrat, F.; Botari, T.; Moudrakovski, I.; Simon, T.; Duppel, V.; Medina, E.; Stolarczyk, J.; Feldmann, J.; Blum, V.; Lotsch, B. V. Urea-Modified Carbon Nitrides: Enhancing Photocatalytic Hydrogen Evolution by Rational Defect Engineering. Adv. Energy Mater. 2017, 1602251.

(48) Reuter, K.; Scheffler, M. Composition, structure, and stability of $\mathrm{RuO} 2(110)$ as a function of oxygen pressure. Phys. Rev. B: Condens. Matter Mater. Phys. 2001, 65, 035406.

(49) Schwarzer, A.; Böhme, U.; Kroke, E. Use of Melem as a Nucleophilic Reagent to Form the Triphthalimide C6N7(phthal)3 New Targets and Prospects. Chem. - Eur. J. 2012, 18, 12052-12058.

(50) Sattler, A.; Schnick, W. On the Formation and Decomposition of the Melonate Ion in Cyanate and Thiocyanate Melts and the Crystal Structure of Potassium Melonate, K3[C6N7(NCN)3]. Eur. J. Inorg. Chem. 2009, 2009, 4972-4981.

(51) Sattler, A.; Pagano, S.; Zeuner, M.; Zurawski, A.; Gunzelmann, D.; Senker, J.; Müller-Buschbaum, K.; Schnick, W. Melamine-Melem Adduct Phases: Investigating the Thermal Condensation of Melamine. Chem. - Eur. J. 2009, 15, 13161-13170.

(52) Lejaeghere, K.; et al. Reproducibility in density functional theory calculations of solids. Science 2016, 351, aad3000.

(53) Blum, V.; Gehrke, R.; Hanke, F.; Havu, P.; Havu, V.; Ren, X.; Reuter, K.; Scheffler, M. Ab initio molecular simulations with numeric atom-centered orbitals. Comput. Phys. Commun. 2009, 180, 21752196.

(54) Havu, V.; Blum, V.; Havu, P.; Scheffler, M. Efficient integration for all-electron electronic structure calculation using numeric basis functions. J. Comput. Phys. 2009, 228, 8367-8379.

(55) Ren, X.; Rinke, P.; Blum, V.; Wieferink, J.; Tkatchenko, A.; Sanfilippo, A.; Reuter, K.; Scheffler, M. Resolution-of-identity approach to Hartree-Fock, hybrid density functionals, RPA, MP2 and GW with numeric atom-centered orbital basis functions. New J. Phys. 2012, 14, 053020.

(56) Knuth, F.; Carbogno, C.; Atalla, V.; Blum, V.; Scheffler, M. Allelectron formalism for total energy strain derivatives and stress tensor components for numeric atom-centered orbitals. Comput. Phys. Commun. 2015, 190, 33-50.

(57) Perdew, J. P.; Burke, K.; Ernzerhof, M. Generalized Gradient Approximation Made Simple. Phys. Rev. Lett. 1996, 77, 3865-3868.

(58) Tkatchenko, A.; Scheffler, M. Accurate Molecular van der Waals Interactions from Ground-State Electron Density and Free-Atom Reference Data. Phys. Rev. Lett. 2009, 102, 073005. 
(59) Rossi, M.; Chutia, S.; Scheffler, M.; Blum, V. Validation challenge of density-functional theory for peptides-example of Ac-PheAla5-LysH(+). J. Phys. Chem. A 2014, 118, 7349-7359.

(60) Schubert, F.; Rossi, M.; Baldauf, C.; Pagel, K.; Warnke, S.; von Helden, G.; Filsinger, F.; Kupser, P.; Meijer, G.; Salwiczek, M.; Koksch, B.; Scheffler, M.; Blum, V. Exploring the conformational preferences of 20-residue peptides in isolation: $\mathrm{Ac}_{-} \mathrm{Ala}_{19}-\mathrm{Lys}+\mathrm{H}^{+}$vs. Ac-Lys- $\mathrm{Ala}_{19}+\mathrm{H}^{+}$and the current reach of DFT. Phys. Chem. Chem. Phys. 2015, 17, 7373-7385.

(61) Tkatchenko, A. Current Understanding of van der Waals Effects in Realistic Materials. Adv. Funct. Mater. 2015, 25, 2054-2061.

(62) Ambrosetti, A.; Reilly, A. M.; DiStasio, R. A., Jr.; Tkatchenko, A. Long-range correlation energy calculated from coupled atomic response functions. J. Chem. Phys. 2014, 140, 18 A508.

(63) Heyd, J.; Scuseria, G. E.; Ernzerhof, M. Erratum: "Hybrid functionals based on a screened Coulomb potential" [J. Chem. Phys. 118, 8207 (2003)]. J. Chem. Phys. 2006, 124, 219906.

(64) Levchenko, S. V.; Ren, X.; Wieferink, J.; Johanni, R.; Rinke, P.; Blum, V.; Scheffler, M. Hybrid functionals for large periodic systems in an all-electron, numeric atom-centered basis framework. Comput. Phys. Commun. 2015, 192, 60-69.

(65) Ihrig, A. C.; Wieferink, J.; Zhang, I. Y.; Ropo, M.; Ren, X.; Rinke, P.; Scheffler, M.; Blum, V. Accurate localized resolution of identity approach for linear-scaling hybrid density functionals and for many-body perturbation theory. New J. Phys. 2015, 17, 093020.

(66) McQuarrie, D. Statistical Mechanics; University Science Books, 2000.

(67) NIST-JANAF Table - Ammonia. http://kinetics.nist.gov/janaf/ html/H-083.html (accessed Sept. 13, 2016).

(68) Chase, M. W. NIST-JANAF Themochemical Tables, 4th ed.; J. Phys. Chem. Ref. Data, 1998; Vol. Monograph 9.

(69) Haar, L.; Gallagher, J. S. Thermodynamic properties of ammonia. J. Phys. Chem. Ref. Data 1978, 7, 635.

(70) Chamorro-Posada, P.; Vázquez-Cabo, J.; Sánchez-Arévalo, F. M.; Martín-Ramos, P.; Martín-Gil, J.; Navas-Gracia, L. M.; Dante, R. C. $2 \mathrm{D}$ to $3 \mathrm{D}$ transition of polymeric carbon nitride nanosheets. J. Solid State Chem. 2014, 219, 232-241.

(71) Seyfarth, L.; Seyfarth, J.; Lotsch, B. V.; Schnick, W.; Senker, J. Tackling the stacking disorder of melon-structure elucidation in a semicrystalline material. Phys. Chem. Chem. Phys. 2010, 12, 22272237.

(72) Dong, H.; Oganov, A. R.; Zhu, Q.; Qian, G.-R. The phase diagram and hardness of carbon nitrides. Sci. Rep. 2015, 5, 9870.

(73) Pickard, C. J.; Salamat, A.; Bojdys, M. J.; Needs, R. J.; McMillan, P. F. Carbon nitride frameworks and dense crystalline polymorphs. Phys. Rev. B: Condens. Matter Mater. Phys. 2016, 94, 094104.

(74) Horvath-Bordon, E.; Riedel, R.; McMillan, P. F.; Kroll, P.; Miehe, G.; van Aken, P. A.; Zerr, A.; Hoppe, P.; Shebanova, O.; McLaren, I.; Lauterbach, S.; Kroke, E.; Boehler, R. High-Pressure Synthesis of Crystalline Carbon Nitride Imide, C2N2(NH). Angew. Chem. Int. Ed. 2007, 46, 1476-1480.

(75) Salamat, A.; Woodhead, K.; McMillan, P. F.; Cabrera, R. Q.; Rahman, A.; Adriaens, D.; Corà, F.; Perrillat, J.-P. Tetrahedrally bonded dense $\mathrm{C}_{2} \mathrm{~N}_{3} \mathrm{H}$ with a defective wurtzite structure: X-ray diffraction and Raman scattering results at high pressure and ambient conditions. Phys. Rev. B: Condens. Matter Mater. Phys. 2009, 80, 104106. 